\title{
Fast-switching printed organic electrochemical transistors including electronic vias through plastic and paper substrates
}

\author{
Jun Kawahara, Peter Andersson Ersman, Kazuya Katoh and Magnus Berggren
}

\section{Linköping University Post Print}

\section{Tweet}

N.B.: When citing this work, cite the original article.

(C)2013 IEEE. Personal use of this material is permitted. However, permission to reprint/republish this material for advertising or promotional purposes or for creating new collective works for resale or redistribution to servers or lists, or to reuse any copyrighted component of this work in other works must be obtained from the IEEE.

Jun Kawahara, Peter Andersson Ersman, Kazuya Katoh and Magnus Berggren, Fastswitching printed organic electrochemical transistors including electronic vias through plastic and paper substrates, 2013, IEEE Transactions on Electron Devices, (60), 6, 2052-2056. http://dx.doi.org/10.1109/TED.2013.2258923

Postprint available at: Linköping University Electronic Press

http://urn.kb.se/resolve?urn=urn:nbn:se:liu:diva-91268 


\title{
Fast-Switching Printed Organic Electrochemical Transistors Including Electronic Vias Through Plastic and Paper Substrates
}

\author{
Jun Kawahara, Peter Andersson Ersman, Kazuya Katoh, Magnus Berggren, Member, IEEE
}

\begin{abstract}
A novel vertical architecture for all-printed organic electrochemical transistors, based on PEDOT:PSS, realized on flexible substrates is reported. The transistors are manufactured along both faces of paper or plastic substrates and via connections are realized using laser ablation and simple punch through using a pin. Successful modulation of the electric current that flows between the two sides of the substrate is achieved using electrolyte-gating and electrochemical modulation of the electronic charge transport of the bulk of the transistor channel. In addition to this, the transistors are exhibiting fast switching and high on/off current ratios.
\end{abstract}

\section{Introduction}

$\mathrm{D}$ uring the last decade the research field of printed electronics (PE) has experienced a tremendous advancement in terms of the performance of single electronic devices, complexity of functional printed systems, and also regarding potential application areas[1-5]. One of the prime key criteria to success with the printed-on-paper and printed-on-plastic electronics revolution is that simple and robust device architectures are developed so that large area, high throughput and low cost manufacturing techniques can be used. Various qualities of flexible plastic foils and cellulose-based papers are explored as the carrying substrates for PE, of which paper nowadays attracts an increased attention in part thanks to its environmentally friendly characteristics[6]. Various sensors[7], thin film transistors (TFTs)[8], displays[9] and supercapacitors[10] have been realized on paper and plastic foils $[8,11,12]$. Moreover, different high volume sheet-based or roll-to-roll printing, coating and lamination tools have been successfully utilized to manufacture complete device systems. However, the device structure of many PE components typically mimics the architecture of devices developed for electronics manufactured using inorganic rigid substrates such as silicon, or thin film devices produced using various evaporation and sputtering methods. Improvements in performance obtained in inorganic-based technologies have in part been achieved by using multi-stack structures[13, 14]. A similar strategy could be utilized also for organic electronic components by constructing device architectures in a vertical configuration; for instance by printing materials on top of each other and to connect devices defined on both sides, using electrical vias, of the carrying flexible substrate to improve integration density and operation performance[15, 16]. Here, we report such an electrical via approach to achieve organic electrochemical transistors (OECT), based on poly(3,4-ethylenedioxythiophene) (PEDOT) doped with poly(styrene sulfonate) (PSS), that exhibit improved drain current switch on/off-ratio and switch time characteristics. The vertical OECTs have been realized on both paper and plastic substrates with electrical vias based on carbon paste. The gate, source, electrolyte and PEDOT:PSS channel are all located on the same side of the substrate, whereas the drain contact is defined on the other side. Current modulation between the source and drain electrodes of these depletion-mode OECTs is obtained by electrochemical switching of the PEDOT:PSS channel. The oxidation state of the PEDOT:PSS channel is controlled by the potential difference between the gate and the source electrodes. A polyelectrolyte, which provides the ions for the two electrochemical half-reactions, separates the PEDOT:PSS-based gate electrode and the transistor channel. Low-voltage operation of the OECTs is possible since electrochemical switching is utilized to define the mode of operation. Also, high drain current levels of the OECTs is possible since electrochemical switching of the channel includes modulation of the electronic conductivity throughout the entire bulk of the channel material, i.e. the transistor operation does not rely on an interface effect of charge accumulation or depletion.

\section{Experimental}

2.1 Materials and equipment

A $50 \mu \mathrm{m}$ thick polyethylene terephthalate (PET) film was used as the plastic substrate for the OECT manufacturing, where the surfaces on both sides are treated to improve the adhesion property (Melinex 454 purchased from DuPont). For OECTs manufactured on a paper, a polyethylene-coated photo paper was provided by Silverdalen Paper Mill (Silver Blade fine paper $120 \mathrm{~g} / \mathrm{m}^{2}$ ). Conducting carbon paste (7102) was purchased from DuPont, PEDOT:PSS inkjet printing ink (Clevios P Jet HC) and PEDOT:PSS screen printing paste (Clevios S V3) were both purchased from Heraeus, and poly(sodium(quaternary ammonium)) was used as the water-based polyelectrolyte, see Figure 1. A PET foil coated with PEDOT:PSS (Orgacon EL-350) was purchased from AGFA. All materials were prepared and used as received without any further purification. Two Keithley 2400 digital source 
measurement units, controlled by a LabView (National Instruments) program, were used for the OECT characterization.
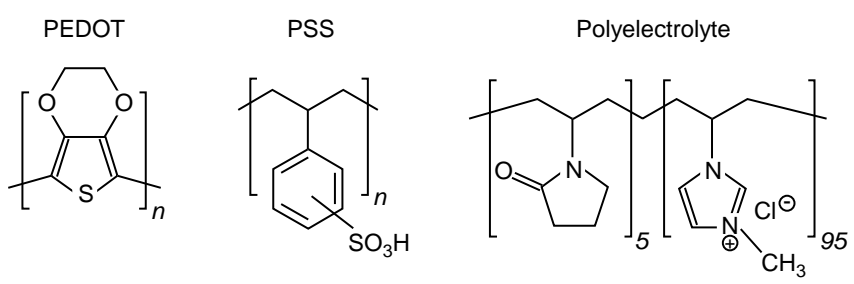

Figure 1. The chemical structures of the materials used in the reported transistor devices; the conducting electrochromic polymer PEDOT (left), its counter polyanion PSS (middle) and the polyelectrolyte (right).

\subsection{Device preparation}

\subsubsection{Plastic substrate}

$\mathrm{A} \mathrm{CO}_{2}$ gas laser drilling machine (YB-HCS03, Panasonic Welding Systems Co. Ltd.) was used to create the holes through the PET substrate The laser was operated at a frequency of $3 \mathrm{kHz}$ with pulse widths of $50 \mu$ s (first pulse) and $25 \mu \mathrm{s}$ (second pulse). The resulting holes through the PET foil has a diameter of about $30 \mu \mathrm{m}$ on the side where the laser beam enters the foil and a diameter of approximately $70 \mu \mathrm{m}$ on the side where the beam escapes the PET foil.

The OECT devices, see Figure 2, are manufactured according to the following process: The carbon paste is manually deposited, using a pipette, into the laser drilled holes to define the electrical conductor through the via holes and as the OECT drain electrode on one side of the substrate. After scraping away excessive carbon paste material from the two surfaces of the substrate, and in order to simplify device probing, a contact for the drain electrode is formed on the backside of the substrate by manually depositing a carbon paste pad with an area of typically several $\mathrm{mm}^{2}$. Immediately after completing the deposition of the carbon paste the substrate is then dried at $120{ }^{\circ} \mathrm{C}$ for 5 minutes.

On the front side of the substrate $100 \times 500 \mu \mathrm{m}^{2}$ rectangles of PEDOT:PSS representing the transistor channels are manufactured using inkjet printing. The rectangles are defined so that each electronic via is entirely covered by one PEDOT:PSS rectangle, see Figure 2. The inkjet printed PEDOT:PSS patterns are then dried at $120{ }^{\circ} \mathrm{C}$ for 5 minutes. The edge of each PEDOT:PSS rectangle, which is oriented away from the drain electrode of the bottom side, is then partly covered by carbon paste to enable safe and simple probing of the source electrode. A 2-3 $\mu \mathrm{L}$ drop of the polyelectrolyte is manually deposited on top of each electronic via and then semi-solidified by drying the substrate for 1 minute at $60{ }^{\circ} \mathrm{C}$. The diameter of each dried electrolyte droplet is about 200-300 $\mu \mathrm{m}$, which thereby ensures that the electrolyte completely covers the electronic via as well as that the electrolyte and the carbon source contact pad are separated by an approximate distance of 100-200 $\mu \mathrm{m}$. The manufacturing of the OECTs are completed by that an Orgacon EL-350 PEDOT:PSS electrode, which serves as the gate electrode, is laminated on top of the electrolyte droplet.

(a)

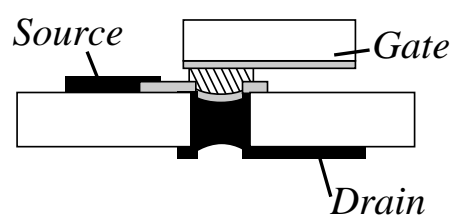

(b)

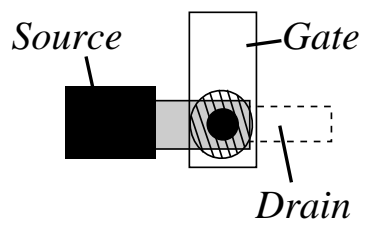

Figure 2. Cross-sectional (a) and top view (b) images of the OECT manufactured on the electronic via substrate. The electrolyte that is in direct contact with the PEDOT:PSS defines the area of the transistor channel. The areas marked in black and gray represents the pattern of conducting carbon and PEDOT:PSS, respectively, while the dashed area represents the electrolyte.

\subsubsection{Paper substrate}

Holes with a diameter of about $200-300 \mu \mathrm{m}$ are generated by mechanically punching a metal pin through the paper substrate. The electronic vias are created using the same carbon paste manufacturing process as described above. The PEDOT:PSS layers that are serving as the OECT channel is now instead screen-printed on the paper substrate. The volume of the electrolyte droplet is increased to approximately $10-15 \mu \mathrm{L}$ due an enlarged diameter of the electronic via. Otherwise, the manufacturing processes are identical to the OECTs manufactured on PET foil, see above.

\subsubsection{Device characterization}

Two Keithley 2400 source measurement units are connected to each other and a LabView (National Instruments) graphical user interface is used to control the transistor measurements and for data recording and storage. The source electrode is connected to ground and the voltage between the drain and the source electrodes $\left(V_{D S}\right)$ is set to $-1 \mathrm{~V}$. Different gate voltages $\left(V_{G}\right)$, ranging from $0 \mathrm{~V}$ to $1 \mathrm{~V}$ or from $0 \mathrm{~V}$ to $1.5 \mathrm{~V}$, are applied to the gate electrode. The electric current levels between the drain and 
source electrodes $\left(I_{D S}\right)$ are continuously recorded while the $V_{G}$ is varied. Hence, the on-to-off and off-to-on switch times of the OECT are measured. The switching times are estimated as the current level reaches $90 \%$ of the averaged maximum OECT current modulation. The time between two consecutive data points in the drain current transient measurements is $5 \mathrm{~ms}$ and five repeated cycles are plotted in the same graph.

If your paper is intended for a conference, please contact your conference editor concerning acceptable word processor formats for your particular conference.

\section{Results and discussions}

The OECTs manufactured on PET substrate were first characterized. The channel of the OECT can be switched off to its non-conducting state within $5 \mathrm{~ms}$, while the time to switch the channel back to its conducting on-state is just below $20 \mathrm{~ms}$, see Figure 3. The electrolyte-gated OECTs described herein operates in the so-called depletion mode, since PEDOT:PSS is highly conducting in its pristine state. Hence, applying a positive $V_{G}$ causes the PEDOT-phase of the channel to reduce to its highly resistive off-state, while setting $V_{G}=0 \mathrm{~V}$ makes PEDOT to re-oxidize to its conducting $o n$-state. The new vertical architecture, here presented, exhibits more than ten times faster off-to-on switching times as compared to a lateral PEDOT:PSS-based OECT device made from identical lateral feature dimensions[5, 17]. OECTs manufactured on a cellulose-based paper substrate was also characterized in a similar manner, see Figure 4. These devices show slightly longer switching times as compared to the OECTs made on PET substrates; $10-20 \mathrm{~ms}$ and 50-60 ms is typically measured for the on-to-off and off-to-on switching time, respectively. One possible explanation to the relatively longer switching time measured for OECTs on paper is that the diameter of the electronic via through the paper substrate is relatively much larger. Hence, the effective volume, i.e. charge capacity, of PEDOT:PSS serving as the transistor channel is then larger for the OECTs on paper. This, in turn, results in a relatively longer time to electrochemically switch the channel from on to off as well as from off to on. An additional explanation is also that the PEDOT:PSS channel is inkjet printed on the PET substrate, while it is screen printed in the case of the paper substrate. This gives that the OECTs manufactured on PET substrates have a relatively thinner channel, which then requires shorter switching time in order to complete the electrochemical reaction throughout the entire bulk of the channel material. The difference in thickness can also explain why different $V_{G}$ needs to be applied in order to reach the same off-current levels for the two OECTs; $V_{G}=1 \mathrm{~V}$ and $V_{G}=1.5 \mathrm{~V}$ was applied to the thin inkjet printed channel (PET foil) and the thick screen printed channel (paper), respectively.
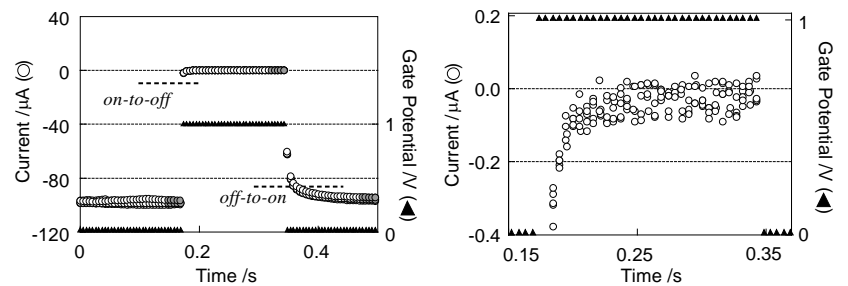

Figure 3. Left: The drain current $I_{D S}$ (open circles) vs. time of the OECT manufactured on a PET substrate is given. The $V_{G}$ (filled triangles) is switched between 0 and $1 \mathrm{~V}$. When the OECT channel is switched from on to off by applying $V_{G}=1 \mathrm{~V}$, the $I_{D S}$ current switches from $100 \mu \mathrm{A}$ to less than $30 \mathrm{nA}$ within $5 \mathrm{~ms}$. When $V_{G}$ is turned off, the $I_{D S}$ switches back to the initial high current level within $20 \mathrm{~ms}$. The gray circles represent the data points used to calculate the average of the current level in the on- and off-state, respectively. The current levels when $I_{D S}$ has reached $90 \%$ of the full modulation are shown in bold dashed lines. Right: the zoom-in plot of the on-to-off switch as $V_{G}$ is switched from $0 \mathrm{~V}$ to $1 \mathrm{~V}$.
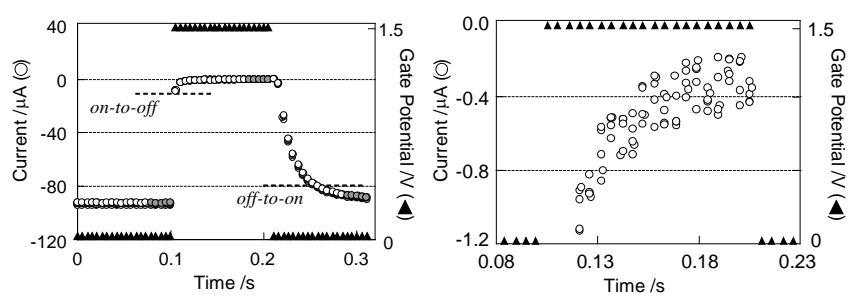

Figure 4. Left: The drain current vs. time characteristics of the OECT printed on a paper is given. Here, the $I_{D S}$ (open circles) is given as a function of time while switching $V_{G}$ (filled triangles) between 0 and $1.5 \mathrm{~V}$. When the OECT channel is switched from on to off, by applying $V_{G}=1.5 \mathrm{~V}$, the $I_{D S}$ current switches from approximately $100 \mu \mathrm{A}$ to $<400 \mathrm{nA}$ within $10-20 \mathrm{~ms}$. When $V_{G}$ is turned off, the $I_{D S}$ switches back to the initial high current level within 50-60 ms. Right: a zoom-in plot of the on-to-off switch when $V_{G}$ is switched from $0 \mathrm{~V}$ to $1.5 \mathrm{~V}$.

The vertical OECT architecture (Figure 5b) described herein provides two prime benefits with respect to the transistor performance as compared to lateral OECTs (Figure 5a); a close to symmetric and fast on-to-off and off-to-on switching characteristics and a simplified manufacturing process. Improved switching characteristics of the vertical OECTs can be 
attributed to two different features; elimination of the so-called reduction front and an effectively shorter transistor channel. The latter implies a decrease of the volume of the PEDOT:PSS material that needs to be switched while operating the OECT. The reduction front represents a detrimental effect on the OECT regarding the off-to-on switching time, while the on-to-off transition typically remains unaffected. The reduction front is established and extends when $V_{D S}$ and $V_{G}$ both are applied for an extensive period of time. This enables migration of the reduced phase of PEDOT far outside the electrolyte edge that supposedly defines the channel. The reduced front of PEDOT propagates towards the negatively biased drain electrode and keeps migrating within the PEDOT:PSS system[18] over a long period of time. Applying a subsequent $V_{G}=0 \mathrm{~V}$ will not result in an immediate re-oxidation of the reduced highly resistive PEDOT phase, which is explained by the high ionic resistivity of the PSS phase as compared to the resistivity of the polyelectrolyte. This effect is common and pronounced in lateral OECT designs based on PEDOT:PSS and causes an off-to-on transition that typically is prolonged by hundreds of milliseconds, and in some cases even seconds[5]. In the OECT design presented here, the carbon paste that serves as the electronic via is sufficiently inert from an electrochemical point of view and is located directly underneath the PEDOT:PSS channel. Hence, there is no excessive PEDOT:PSS along the drain electrode that can undergo reduction and therefore no reduction front can propagate towards the drain electrode. This, in turn, results in equally fast on-to-off and off-to-on switching times[xx].

(a)

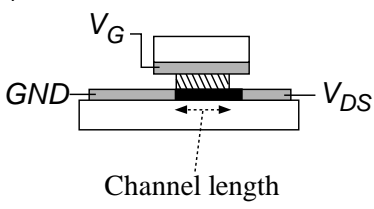

(b)

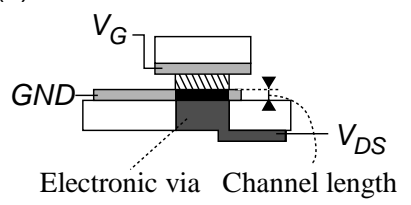

$\square$ Substrate $\square$ PEDOT:PSS $\mathbb{\mathbb { N } \text { Electrolyte }}$

Conducting carbon Reduced PEDOT:PSS

Figure 5. Cross-sectional illustrations showing the difference between the OECT channel lengths for (a) the lateral OECT design and (b) the vertical OECT design, where the channel length of the latter equals the thickness of the deposited PEDOT:PSS. The reduction front is illustrated in the lateral OECT design (a) by the reduced area of PEDOT:PSS that is propagating outside the electrolyte edge towards the drain electrode.

In lateral OECT architectures the transistor channel corresponds to the bulk of the PEDOT:PSS thin film located between the source and drain electrodes and that is covered by the electrolyte (cf. Figure 5a, References [5] and [17]). The channel thickness, length and width are all limited by the patterning resolution of the deposition techniques utilized; typically the lateral resolution exceeds tens of micrometers for common printing techniques. The total volume of the PEDOT phase in the transistor channel determines the total charge that is required to switch the OECT on and off. This charge capacity, together with the impedance characteristics for both mobile ions in the electrolyte and for ions and holes in PEDOT:PSS, govern the switching time of the OECT. For the lateral OECTs, the strategy to shorten the switching time would then be to minimize the channel volume by improving the resolution of the printing techniques and to improve the charge conductivity of the electrolyte and the PEDOT:PSS materials systems. The vertical OECT structure, reported here, has a carbon-based electronic via and drain electrode located directly underneath the PEDOT:PSS transistor channel, see Figure 5b. This vertical channel configuration provides a much shorter OECT channel length that simply is defined as the thickness of the inkjet printed PEDOT:PSS layer. This thickness is typically around a few $100 \mathrm{~nm}$, in some cases even thinner. The lateral dimension of the electrolyte alone effectively defines the width of the channel. From a manufacturing point of view, it is therefore an easier task to improve the transistor performance if the OECT channel is defined only by the resolution of the deposited electrolyte layer, while the resolution of the PEDOT:PSS deposition becomes less important. In this report the electrolyte drop was manually deposited. The most straightforward route to shrink the volume of PEDOT:PSS, and thereby improve switching time and on/off-ratio even further, would be to utilize a high-resolution deposition technique for the electrolyte, such as screen printing. Furthermore, in the vertical OECT the PEDOT:PSS channel is deposited on top of the carbon via. The surface of this via is typically not smooth and the surface roughness is expected to be on the same order as the thickness of the PEDOT:PSS film thickness. Since gating is carried out through an electrolyte, and that current modulation is achieved throughout the entire bulk of the channel, no requirements for ultra-smooth surfaces are required. The PEDOT:PSS-based OECT technology is robust, which make this vertical device configuration possible.

The obtained on/off-ratio of the $I_{D S}$ exceeds 4000 (approximately $98 \mu \mathrm{A}$ (on) vs. $23 \mathrm{nA}$ (off)) for the OECT PET substrate, while it reaches 280 (approximately $90 \mu \mathrm{A}$ (on) vs. $320 \mathrm{nA}$ (off)) for the OECT on top of the paper substrate. Especially, the former on/off-ratio is expected to be sufficiently high to operate electrochromic active matrix addressed displays without any drastic cross-talk effects. The latter on/off-ratio is too low for such applications but still promising in printed organic logic circuits. The on-current levels is about the same for both vertical OECT devices, independent of the substrate used, which is explained by the intrinsic conduction property of the PEDOT:PSS channel material. 


\section{Conclusion}

Printed vertical electrochemical transistors resulting in fast switching and high on/off current ratios are demonstrated. The transistor devices are gated via a polyelectrolyte and utilize both sides of the plastic or paper substrate. An electronic via that also defines the channel makes connection between the two sides possible. Such device concept is potentially useful for integrated electronic systems for three dimensional printed electronic subcomponents and systems. For instance, in printed active matrix addressed displays sufficiently short switching time and low leakage current are two criterions that are required in order to obtain proper operation with short display update time and minor cross-talk between neighboring pixels. The devices presented in this report therefore represent a promising concept for establishing printed organic matrix-addressed displays with high fill factor and transistor circuitry that connects two sides of for instance printed electronic labels.

\section{Acknowledgements}

Prime funding for this project was provided by Lintec Corporation. Also, the authors wish to thank VINNOVA (Printed Electronics Arena), KAW (Power Papers) and Önnesjö Foundation for additional financial support.

\section{References}

[1] F. C. Krebs, J. Fyenbo, and M. Jørgensen, "Product integration of compact roll-to-roll processed polymer solar cell modules: Methods and manufacture using flexographic printing, slot-die coating and rotary screen printing," Journal of Materials Chemistry, vol. 20, pp. 8994-9001, 2010.

[2] G. H. Gelinck, H. E. A. Huitema, E. V. Veenendaal, E. Cantatore, L. Schrijnemakers, J. B. P. H. Van Der Putten, T. C. T. Geuns, M. Beenhakkers, J. B. Giesbers, B. H. Huisman, E. J. Meijer, E. M. Benito, F. J. Touwslager, A. W. Marsman, B. J. E. Van Rens, and D. M. De Leeuw, "Flexible active-matrix displays and shift registers based on solution-processed organic transistors," Nature Materials, vol. 3, pp. 106-110, 2004.

[3] A. C. Arias, S. E. Ready, R. Lujan, W. S. Wong, K. E. Paul, A. Salleo, M. L. Chabinyc, R. Apte, R. A. Street, Y. Wu, P. Liu, and B. Ong, "All jet-printed polymer thin-film transistor active-matrix backplanes," Applied Physics Letters, vol. 85, pp. 3304-3306, 2004.

[4] V. Subramanian, J. M. J. Fréchet, P. C. Chang, D. C. Huang, J. B. Lee, S. E. Molesa, A. R. Murphy, D. R. Redinger, and S. K. Volkman, "Progress toward development of all-printed RFID tags: Materials, processes, and devices," Proceedings of the IEEE, vol. 93, pp. 1330-1338, 2005.

[5] P. Andersson, R. Forchheimer, P. Tehrani, and M. Berggren, "Printable all-organic electrochromic active-matrix displays," Advanced Functional Materials, vol. 17, pp. 3074-3082, 2007.

[6] D. Tobjörk and R. Österbacka, "Paper electronics," Advanced Materials, vol. 23, pp. 1935-1961, 2011.

[7] J. Sarfraz, D. Tobjork, R. Osterbacka, and M. Linden, "Low-cost hydrogen sulfide gas sensor on paper substrates: Fabrication and demonstration," IEEE Sensors Journal, vol. 12, pp. 1973-1978, 2012.

[8] R. Bollström, A. Määttänen, D. Tobjörk, P. Ihalainen, N. Kaihovirta, R. Österbacka, J. Peltonen, and M. Toivakka, "A multilayer coated fiber-based substrate suitable for printed functionality," Organic Electronics: physics, materials, applications, vol. 10, pp. 1020-1023, 2009.

[9] P. Andersson, D. Nilsson, P. O. Svensson, M. Chen, A. Malmström, T. Remonen, T. Kugler, and M. Berggren, "Active matrix displays based on all-organic electrochemical smart pixels printed on paper," Advanced Materials, vol. 14, pp. 1460-1464, 2002.

[10] L. Yuan, X. Xiao, T. Ding, J. Zhong, X. Zhang, Y. Shen, B. Hu, Y. Huang, J. Zhou, and Z. L. Wang, "Paper-based supercapacitors for self-powered nanosystems," Angewandte Chemie - International Edition, vol. 51, pp. 4934-4938, 2012.

[11] A. Russo, B. Y. Ahn, J. J. Adams, E. B. Duoss, J. T. Bernhard, and J. A. Lewis, "Pen-on-paper flexible electronics," Advanced Materials, vol. 23, pp. 3426-3430, 2011.

[12] P. Ihalainen, A. Määttänen, J. Järnström, D. Tobjörk, R. Österbacka, and J. Peltonen, "Influence of surface properties of coated papers on printed electronics," Industrial and Engineering Chemistry Research, vol. 51, pp. 6025-6036, 2012.

[13] H. H. S. Lee and K. Chakrabarty, "Test challenges for 3D integrated circuits," IEEE Design and Test of Computers, vol. 26, pp. 26-35, 2009.

[14] K. Banerjee, S. J. Souri, P. Kapur, and K. C. Saraswat, "3-D ICs: A novel chip design for improving deep-submieroraeter interconnect performance and systems-on-chip integration and systems-on-chlp integration," Proceedings of the IEEE, vol. 89, pp. 602-632, 2001

[15] S. Song, B. Cho, T. W. Kim, Y. Ji, M. Jo, G. Wang, M. Choe, Y. H. Kahng, H. Hwang, and T. Lee, "Three-dimensional integration of organic resistive memory devices," Advanced Materials, vol. 22, pp. 5048-5052, 2010.

[16] J. Liu, L. Herlogsson, A. Sawatdee, P. Favia, M. Sandberg, X. Crispin, I. Engquist, and M. Berggren, "Vertical polyelectrolyte-gated organic field-effect transistors," Applied Physics Letters, vol. 97, 2010.

[17] J. Kawahara, P. Andersson Ersman, D. Nilsson, K. Katoh, Y. Nakata, M. Sandberg, M. Nilsson, G. Gustafsson, and M. Berggren, "Flexible active matrix addressed displays manufactured by printing and coating techniques," Journal of Polymer Science Part B: Polymer Physics, pp. n/a-n/a, 2012.

[18] T. Johansson, N. K. Persson, and O. Inganäs, "Moving Redox Fronts in Conjugated Polymers Studies from Lateral Electrochemistry in Polythiophenes," Journal of the Electrochemical Society, vol. 151, pp. E119-E124, 2004. 
